\title{
Review
}

\section{Electrophysiology of Basal Ganglia and Cortex in Models of Parkinson Disease}

\author{
Damien J. Ellens and Daniel K. Leventhal* \\ Department of Neurology, University of Michigan Medical School, MI, USA
}

\begin{abstract}
Incomplete understanding of the systems-level pathophysiology of Parkinson Disease (PD) remains a significant barrier to improving its treatment. Substantial progress has been made, however, due to the availability of neurotoxins that selectively target monoaminergic (in particular, dopaminergic) neurons. This review discusses the in vivo electrophysiology of basal ganglia (BG), thalamic, and cortical regions after dopamine-depleting lesions. These include firing rate changes, neuronal burst-firing, neuronal oscillations, and neuronal synchrony that result from a combination of local microanatomic changes and network-level interactions. While much is known of the clinical and electrophysiological phenomenology of dopamine loss, a critical gap in our conception of PD pathophysiology is the link between them. We discuss potential mechanisms by which these systems-level electrophysiological changes may emerge, as well as how they may relate to clinical parkinsonism. Proposals for an updated understanding of BG function are reviewed, with an emphasis on how emerging frameworks will guide future research into the pathophysiology and treatment of PD.
\end{abstract}

Keywords: Parkinson disease, electrophysiology, 1-Methyl-4-phenyl-1, 2, 3, 6-tetrahydropyridine, models, animal, dopamine

\section{INTRODUCTION}

Though Parkinson Disease (PD) is a multifaceted neuropsychiatric syndrome, its defining characteristics are the motor features of progressive rigidity, bradykinesia, postural instability, and rest tremor. The best-studied pathological finding is dopaminergic cell loss in the substantia nigra, pars compacta (SNc), which projects primarily to the striatum. Because dopamine replacement therapy (DRT) is universally effective for bradykinesia and rigidity (and usually effective for tremor), dopamine loss must be causally related to motor impairment. The chain of events from dopamine loss to motor disability, however, remains unclear. To optimize effectiveness and minimize complications of PD treatments, it is essential to understand how they influence brain networks involved in the pathophysiology of PD.

${ }^{*}$ Correspondence to: Daniel K. Leventhal, 4027 BSRB, 109 Zina Pitcher Place, Department of Neurology, University of Michigan Medical School, Ann Arbor, MI 48109-2200, USA. Tel.: + 1734764 7867; Fax: +1 734998 2388; E-mail: dleventh@med.umich.edu.
Over the last 30 years, great strides have been made in understanding the network physiology of corticalbasal ganglia (BG)-thalamic circuits in health and disease. Critical to this effort have been animal models of PD, which recapitulate many motor and electrophysiological features of human disease. Here, we review physiological findings in animal models of dopamine depletion, their behavioral correlates, and implications for PD treatment and future research.

\section{ANIMAL MODELS}

There are multiple animal models of PD, but the vast majority of electrophysiological data come from use of the monoaminergic neuron-selective toxins 6hydroxydopamine (6-OHDA) and 1-methyl-4-phenyl1,2,3,6-tetrahydropyridine (MPTP).

6-OHDA moves into neurons via monoamine transporters, where it undergoes oxidation to toxic free radicals [1]. Because it does not cross the blood-brain barrier, it is infused directly into the brain. If targeted to the medial forebrain bundle, nearly complete 
dopamine denervation of the ipsilateral hemisphere is achieved. It can also be injected into monoaminergic terminal regions, killing neurons projecting into those areas [2]. This may confine dopamine loss to specific nuclei, though dopaminergic cell bodies often send collaterals to multiple regions. An important consideration is the time-course of neuronal death, which may progress over 2 weeks or longer [3].

The MPTP model emerged after IV drug users inadvertently synthesized MPTP attempting to make meperidine, and developed acute parkinsonism [1]. MPTP produces dopa-responsive parkinsonism in primates, and causes severe monoaminergic depletion in mice (but not rats [2]). MPTP can be administered systemically, or, in primates, intracarotid infusions can be used to create hemiparkinsonism. MPTP treated animals demonstrate regional differences in striatal dopaminergic denervation, dopaminergic cell death, and noradrenergic cell death similar to PD. Some primate species (notably African Green monkeys) develop a classic rest tremor, while others (notably macaques) develop an intermittent higher frequency postural/action tremor [4].

\section{FUNCTIONAL ANATOMY}

The BG are deep gray matter structures that form recurrent loops with the cortex and thalamus. In "standard" models, the striatum is the sole input nucleus (Fig. 1A), though newer models also incorporate the hyperdirect cortical-subthalamic nucleus (STN) pathway (Fig. 1B) [5]. Globus pallidus, pars externa (GPe) is essentially isolated from extra-BG structures, and the output nuclei comprise the globus pallidus, pars interna (GPi; entopeduncular nucleus, EPN, in rodents) and substantia nigra, pars reticulata $(\mathrm{SNr})$. $\mathrm{SNc}$ modulates cortical-BG-thalamic circuits with dopaminergic innervation of the striatum principally, but also other BG nuclei, frontal cortex, and thalamus.

\section{The striatum}

The striatum receives glutamatergic input from widespread areas of cortex [6], as well as thalamic intralaminar (centromedian/parafascicular -CM/Pf) and BG-recipient relay nuclei [7]. GABAergic inputs come from the GPe [8], and it sends GABAergic efferents to GPe and the BG output nuclei.

The striatum is composed of GABAergic medium spiny projection neurons (MSNs) and several interneuron types in two interlaced compartments known as "patches" (also called striosomes) and "matrix" [9].
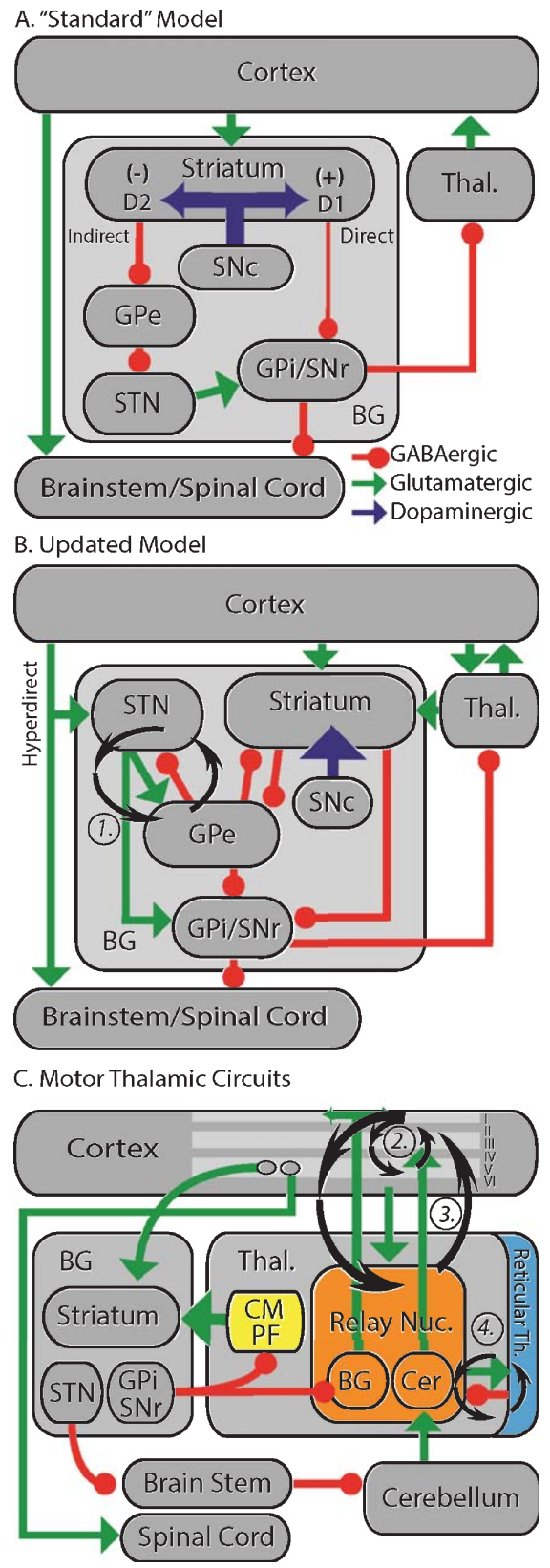

Fig. 1. Cortical-BG-thalamic models (see Functional Anatomy for full descriptions). (A) The "standard" model. Green/light gray arrows-excitatory (glutamatergic) projections; red/black circles-inhibitory (GABAergic) projections; blue/dark gray arrows - dopaminergic projections. (+) and (-) indicate excitatory and inhibitory effects of dopamine on MSNs, respectively. Abbreviations are defined in the text. (B) Updated model. Not all connections described in the text are illustrated for clarity. (C) Model emphasizing thalamic circuits. Roman numerals indicate cortical layers. Ovals in layer V indicate distinct populations of IT and PT neurons. Note the distinct projection patterns of the cerebellar and BG-recipient thalamus. In the thalamus, "BG" and "Cer" indicate the BG and cerebellar-recipient regions, respectively. Circular arrows in (B) and (C) indicate candidate oscillation generators. 
MSNs represent 90-95\% of neurons depending on the species, with lower percentages in primates $[10,11]$. They fire sparsely, requiring coordinated excitatory synaptic input to initiate spiking [12]. They are divided into two groups of roughly equal proportions based on projection patterns [13]. The monosynaptic connection from striatum to GPi/SNr is termed the "direct" pathway, while "indirect" pathway MSNs (iMSNs) synapse in the GPe. Direct pathway MSNs (dMSNs) also send collaterals to the GPe. dMSNs express low affinity D1 dopamine receptors, while iMSNs express high affinity D2 dopamine receptors [13]. Phasic increases or decreases in striatal dopamine concentration therefore likely have distinct effects on the direct and indirect pathways.

There are at least three subtypes of striatal GABAergic interneurons. Parvalbumin-positive fast spiking interneurons (FSIs) selectively and powerfully inhibit MSNs [11], though their precise role in striatal microcircuitry remains to be determined [14]. The physiology of other GABAergic interneurons (nitric-oxide synthase and calretinin-positive) is less well-studied [11].

Cholinergic interneurons probably correspond to tonically active neurons (TANs) recorded in vivo, which fire in a regular pattern below $10 \mathrm{~Hz}$ [15]. Their axons arborize densely and diffusely in the striatal matrix, with sparse crossings into the patch compartment $[16,17]$. Their firing patterns are correlated even in awake, behaving animals, and may therefore transmit coordinated signals to large striatal regions. Striatal cholinergic signaling directly influences interneuron and MSN activity, and also interacts with dopamine to regulate synaptic plasticity [13]. The "TAN pause" is a transient, coordinated decrease in TAN firing in response to salient behavioral events that is dependent on afferents from CM/Pf. Of relevance to PD, the TAN pause is lost after striatal dopamine depletion [15].

Dopamine modulates striatal output on multiple timescales. D1 receptor stimulation acutely enhances MSN excitability, while D2 receptor binding has opposite effects [13]. Dopamine also influences synaptic plasticity at cortico- and thalamostriatal synapses onto dMSNs and iMSNs in distinct ways [13, 18]. Striatal interneurons express multiple dopamine receptor subtypes; their responses to dopamine are therefore difficult to predict. Furthermore, chronic dopamine loss changes striatal microanatomy. Dendritic spines and glutamatergic synapses onto iMSNs are greatly diminished [19], though dMSN excitability is diminished and iMSN excitability enhanced to cortical stimulation [20]. This may be partially explained by strengthen- ing of remaining corticostriatal synapses [21]. FSIs, which under normal circumstances have a slight preferential innervation of dMSNs compared to iMSNs, become much more strongly connected to iMSNs after dopamine loss [22].

\section{The subthalamic nucleus}

Glutamatergic input to STN comes from frontal regions including primary, pre-, and supplementary motor cortex (Fig. 1B) [5]. Additional excitatory input comes from CM/Pf [7], which may give the STN access to ascending "salience" signals from the brainstem [23]. GABAergic input is primarily from the GPe [24], and sparse dopaminergic afferents come from SNc [25]. The STN projects to GPe, forming a GPeSTN loop that is strengthened after dopamine depletion (Fig. 1B, circuit 1) [26]. STN also projects to the output nuclei, where it synapses throughout the dendritic tree. More recently, a disynaptic projection to the cerebellar cortex via the brainstem has been identified (Fig. 1C) [27]. STN projections diverge to innervate large regions of the $\mathrm{BG}$, suggesting that it cannot encode detailed information in population activity, but may broadcast information-poor timing signals. Principal STN neurons are spontaneously active, with tonic in vivo firing rates in the $10-30 \mathrm{~Hz}$ range in nonhuman primates [28]. They express T-type (Cav 3) calcium channels that render them prone to "rebound" bursting, and the likelihood of bursting may be modulated by subthalamic dopamine [25].

\section{Globus pallidus, pars externa}

GPe (simply GP in rodents) receives GABAergic afferents from striatal iMSNs and glutamatergic input from the STN and CM/Pf [7]. Distinct populations of GPe neurons project to striatum and downstream nuclei (STN, GPi, and SNr) [8], where they typically form large perisomatic synapses. This anatomy suggests a central role for the GPe in coordinating BG-wide activity (Fig. 1B).

In vivo recordings have consistently found that most GPe cells fire at high rates with occasional pauses, and another group tends to fire sparsely with intermittent bursts [29]. External pallidal neurons can also be classified based on their synchronization to local field potentials (LFPs) [8], but it is not known whether these two classification schemes are related to each other. At least in dopamine-depleted rats, the LFP-based classification identifies populations that project uniquely to striatum or STN/GPi/SNr. 
Dopaminergic projections from SNc have pre- and post-synaptic effects in GPe, though the dopaminergic innervation is much weaker than in striatum [25]. How dopamine influences pallidal activity in vivo is not well understood, though local lesioning of dopamine terminals recapitulates some features of PD [30]. As in the striatum and STN, chronic dopamine loss changes GPe anatomy and physiology. The intrinsic pacemaking ability of pallidal neurons is lost [31], intrapallidal collaterals are strengthened [32], and subthalamopallidal connections are strengthened [26].

\section{The output nuclei (GPi and SNr)}

The BG output nuclei receive afferents primarily from dMSNs, GPe, and STN, though afferents from $\mathrm{CM} / \mathrm{Pf}$ also exist [7]. Striatal afferents tend to synapse distally, pallidal afferents proximally, and subthalamic afferents throughout the dendritic tree [33, 34]. There is also dopaminergic input to both nuclei, with a unique dendritic release mechanism in $\mathrm{SNr}$ [25]. GPi projects primarily to the motor relay nuclei of the thalamus, as well as CM/Pf and the pedunculopontine nucleus (PPN) [35]. The SNr has similar projections, but also sends efferents to the superior colliculus (SC) and SNc [34]. It is therefore associated with orienting movements and saccades, as well as regulating nigrostriatal dopaminergic neurons.

BG output neurons exhibit spontaneous activity at high firing rates [28] during quiet wakefulness, but without the pauses that characterize most GPe neurons. These neurons are capable of bursting at very high rates, which is enhanced after dopamine depletion $[28,36]$. As in the GPe and STN, enhanced bursting in the dopamine-depleted state may depend on both altered afferent activity and changes intrinsic to the output nuclei [37].

\section{Substantia nigra, pars compacta}

The principal dopaminergic innervation of $\mathrm{BG}$ motor and associative circuits comes from SNc [38]. Over $70 \%$ of SNc input is GABAergic, including afferents from the rostromedial tegmental nucleus (RMTg) [39], striatal patches [40] and $\mathrm{SNr}$ [41]. It also receives glutamatergic afferents from diverse subcortical structures including STN and PPN [38]. Further, local somatodendritic dopamine release influences both $\mathrm{SNc}$ and $\mathrm{SNr}$ [38]. The principal target of nigral dopamine is the striatum, though there are also projections to frontal cortex, the other BG nuclei, and thalamus. SNc neurons have intrinsic pacemaker activity, firing below $10 \mathrm{~Hz}$ in vivo to maintain tonic dopamine levels in the striatum (and other targets). Phasic changes in nigrostriatal signaling, tightly regulated by upstream structures (e.g., lateral habenula via RMTg [39]) are believed to be critical for striatum-based implicit learning processes [42].

\section{Thalamic interactions with the $B G$}

BG afferents form large GABAergic perisomatic synapses in thalamic relay nuclei [43], which are relatively unique in that "driver" inputs to other thalamic nuclei are glutamatergic [44]. These relay nuclei form recurrent loops with cortex believed to generate oscillatory brain rhythms [45]. Cortex and thalamic relays send collaterals to the reticular thalamus, which in turn projects back to thalamic relays (Fig. 1C). Two thalamocortical projection patterns are recognized. "Matrix" neurons tend to project diffusely to cortical layer I, while "core" neurons have more focused projections to layers III-IV. BG-receiving areas consist primarily of matrix-like cells, while cerebellar recipient regions consist of core-like cells [46]. Thalamocortical neurons express T-type ( $\mathrm{Cav} 3$ ) calcium channels that allow transitions from tonic to burst-firing modes [45] in response to hyperpolarization. Modeling studies [47] suggest that tonic firing allows faithful transmission of afferent signals to cortex, while bursting modes may inhibit cortico-cortical communication. Therefore, the BG may modulate information flow in other circuits, rather than transmitting information from the $\mathrm{BG}$ to cortex [47].

$\mathrm{CM} / \mathrm{Pf}$ receives dense afferents from brainstem (e.g., PPN and SC) and BG output nuclei [7], as well as cortical afferents. It projects primarily to striatum, where it synapses on MSNs, GABAergic interneurons, and cholinergic interneurons (Fig. 1C). It also has weaker projections to cortex, STN, GPi, and SNr. CM/Pf degenerates in PD and the MPTP model of parkinsonism [48], potentially contributing to the clinical and physiological features of PD.

\section{Cortex}

A comprehensive review of cortical anatomy and physiology is beyond the scope of this review, but a few key points should be emphasized. First, corticostriatal projections originate in all areas of cortex [6], but corticosubthalamic projections originate in frontal areas [5]. Second, cortical efferents can be broadly classified as intratelencephalic (IT) and pyramidal tract-like (PT) neurons (Fig. 1C) [49]. IT neurons form slow conducting corticocortical and corticostriatal pathways 
that project bilaterally (e.g., from primary motor cortex) or ipsilaterally (e.g., from primary somatosensory cortex), and receive minimal direct input from the BG-recipient relay thalamus [50]. PT neurons send at most weak collaterals to the ipsilateral striatum, receive strong inputs from the BG-recipient relay thalamus, and may provide corticosubthalamic innervation [6]. Thus, the "closed loop" architecture of cortico-BGthalamic circuits may not be entirely closed.

\section{ELECTROPHYSIOLOGICAL CHANGES IN ANIMAL MODELS OF PD}

Electrophysiological phenomena associated with dopamine loss include changes in firing rates, burst activity, neuronal oscillations, and synchrony.

\section{Rate changes}

According to the prevailing "rate" model of BG physiology (Fig. 1A) [51], striatal dopamine increases dMSN firing, decreases iMSN firing, and, ultimately, suppresses BG output to release thalamocortical circuits from tonic inhibition. In support of this model, lesions [52] or transient inactivation [53] of the overactive STN in MPTP-treated monkeys improves parkinsonism. More persuasively, selective activation of dMSNs or iMSNs using optogenetic techniques dramatically alters locomotor activity in a manner consistent with the rate model [54]. Despite great predictive value, however, the rate model is not consistent with all observations. It predicts that decreased BG output should lead to increased movement, but there are many distinct hyperkinetic movement disorders. Furthermore, GPi lesions improve dystonia, chorea, and levodopa induced dyskinesias, all of which should worsen in the standard model.

For the most part, chronic dopamine depletion leads to changes in the firing rates of BG neurons in awake, behaving animals that match rate model predictions. In MPTP treated monkeys [28, 36, 50, 55], firing rates have been found to increase in $\mathrm{STN}$ and $\mathrm{GPi} / \mathrm{SNr}$, but decrease in GPe, thalamus, and motor cortex. The cortical firing rate decrease may be specific to PT neurons [50]. In anesthetized rats, dMSNs and iMSNs decrease and increase their firing rates, respectively, after 6-OHDA lesions [20,56]. Furthermore, firing rate changes after chronic levodopa [57], dopamine agonist [58], or combined [59] treatment in MPTP-treated monkeys are consistent with the standard model. Not all studies support the rate model, however. Some show no or inconsistent changes in firing rates after MPTP despite clinical parkinsonism [53, 55, 60-63]. Also, STN deep brain stimulation (DBS) at therapeutic frequencies increases GPi firing rates in parkinsonian monkeys despite clinical improvement [64].

\section{Burst firing}

Bursts may be defined as brief episodes of high frequency firing against slower background activity. For many neurons, bursting is a distinct firing mode supported by specific ion channels, not simply a transient firing rate increase. Identifying firing mode transitions requires intracellular recordings, however, which are technically difficult in behaving animals [65]. The cellular mechanisms underlying extracellularly recorded parkinsonian "bursts" cannot, therefore, be established definitively, and likely differ across regions.

\section{Burst-firing in GPe, STN, and GPi}

Several studies in the primate MPTP and rodent 6OHDA models have found enhanced bursting in GPe, STN, and GPi/SNr (Fig. 2A) [28, 36, 53, 63, 66-68]. Bursting is reduced by IV levodopa [53] or chronic dopamine agonist/levodopa combination therapy [59], suggesting a relationship between burst-firing and clinical parkinsonism. In humans treated with apomorphine, however, STN and GPi bursting often increase compared to the "off" state, though many subjects became dyskinetic [69-71]. Therapeutic, but not subtherapeutic, GPe [72] and STN [64] DBS reduce GPi bursting. Conversely, low frequency STN DBS, which may exacerbate parkinsonism, increases GPi bursting [73]. In humans, effective GPi DBS reduced GPi burst-firing [74], though conflicting results were obtained in MPTPtreated monkeys [75]. Overall, it seems that burst-firing along the indirect pathway increases after dopamine depletion and decreases with effective therapy, though there is not uniform agreement across experiments.

\section{Thalamic bursts}

There are limited data comparing thalamic activity between dopamine-depleted and intact subjects. In one study comparing thalamic activity before and after MPTP, a non-significant increase was found in the proportion of burst-firing neurons in the BG-recipient thalamus. Bursting was significantly increased, however, in the cerebellar-recipient thalamus [62]. Others have found that clinically effective STN $[73,76]$ or GPe [72] DBS is associated with decreased BG-recipient thalamic bursting compared to the "off" state. Results in the cerebellar recipient thalamus are less consistent $[73,76]$. Human studies indicate that thalamic bursting 


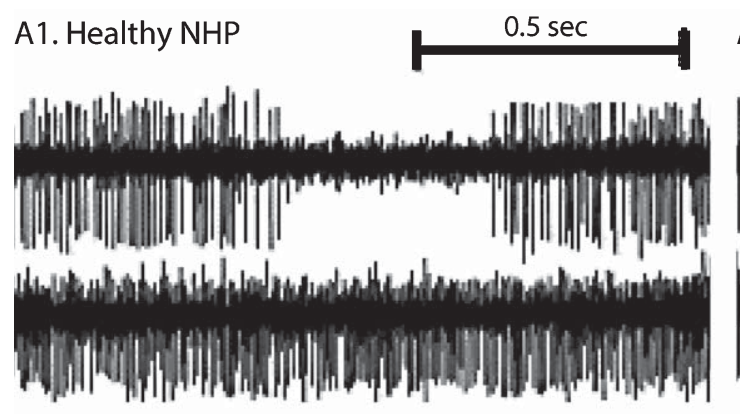

A2. Dopamine (-) NHP

B1. Healthy Rat B2. Dopamine (-) Rat

C. Dopamine (-) NHP

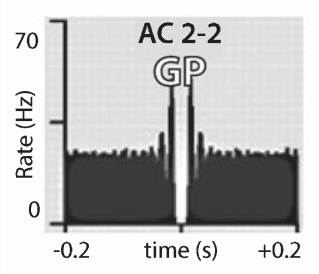

D1. On Levodopa

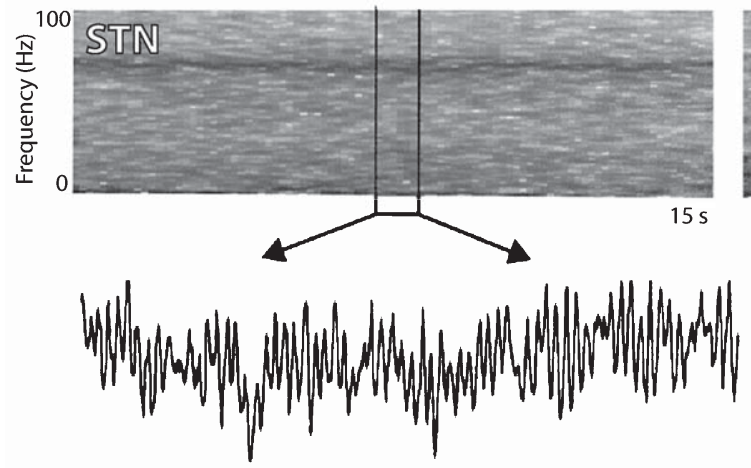

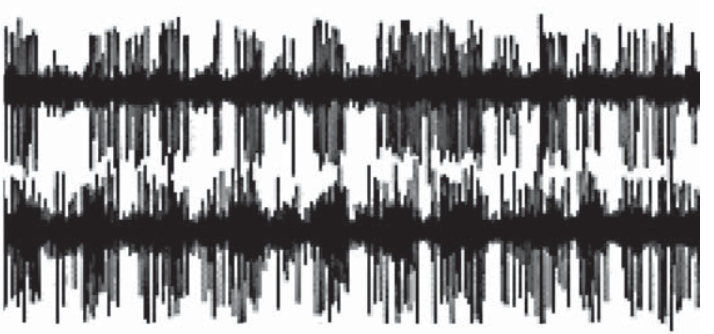

Fig. 2. Electrophysiological phenomena observed in parkinsonian subjects. Single unit pallidal recordings from dopamine-intact (A1) and MPTP-treated (A2) non-human primates (NHP). The upper trace in A1 shows a typical GPe "high-frequency discharge with pauses" pattern. Pallidal burst-firing is increased after MPTP treatment [104]. (B) Auto- and cross-correlograms of single unit activity from anesthetized rats during "cortical activation," emphasizing the emergence of synchronized beta oscillations. AC-autocorrelogram, CC-cross-correlogram. Numbers identify individual units (for example CC 2-3 is the cross-correlogram between units 2 and 3 [92]). (C) Cross-correlogram illustrating non-oscillatory synchrony among GPe neurons in an MPTP-treated NHP [59]. The ordinate displays the mean-subtracted (MS) firing rate. (D) Time-frequency plots and sample LFPs from the STN of a patient with PD on (D1) and off (D2) levodopa [89]. (Figures adapted with permission from the relevant sources).

is common in PD [77, 78], but lack healthy controls. Because these are all extracellular recordings, it is uncertain whether they reflect true low-threshold spike bursts mediated by T-type $\mathrm{Ca}^{2+}$ channels, or brief periods of rapid tonic firing [65]. Excessive thalamic relay burst-firing is probably characteristic of PD, though the evidence is less robust than along the indirect pathway.

\section{Cortical bursts}

Dopamine depletion increases burst-firing in primary motor cortex as measured by the proportion of bursty neurons [61], as well as the percentage of time spent in bursts [50]. As for firing rate, bursting increases among PT, but not IT, neurons [50]. Since thalamic BG-recipient relay nuclei project preferentially to PT neurons, these data suggest that cortical bursting is driven by the thalamus. On the other hand, the long duration of cortical bursts (greater than $1 \mathrm{~s}$ ) is quite different from the brief bursts observed in the $\mathrm{BG}$ and thalamus.

\section{Striatal bursts}

A single study compared MSN bursting between awake, behaving control and 6-OHDA-lesioned rats, 
finding increased burstiness after 6-OHDA lesions [79]. Given the low intraburst firing rate $(\sim 25 \mathrm{~Hz})$, and different set of ion channels expressed on MSNs, the physiology of striatal bursts is probably different from pallidal, subthalamic, or thalamic bursting.

\section{Neuronal oscillations}

"Oscillatory activity" is a nonspecific term indicating that some measure of neuronal function repeats itself periodically. This may be reflected in single unit spiking or local field potentials (LFPs) (Fig. 2B, D). LFPs are generally easier to measure than single unit activity, and are accessible with large, low impedance electrodes. They are believed to represent the summation of local transmembrane currents and synaptic activity, and may therefore reflect afferent activity.

In vivo recordings have demonstrated exaggerated single unit oscillations at tremor frequencies and harmonics, though the relationship between neuronal and mechanical oscillations is complex. Bimodal distributions ( $\sim 5$ and $10 \mathrm{~Hz}$ peaks) of single unit oscillation frequencies are found in the GPi and STN of tremulous MPTP-treated monkeys [4, 28, 60]. TAN firing also becomes oscillatory after MPTP [80]. Interestingly, higher frequency oscillations at $10-15 \mathrm{~Hz}$ may correlate best with the typical $5 \mathrm{~Hz}$ rest tremor of PD [4] (but see [53]). Further, inactivation of the STN in tremulous monkeys eliminates tremor and $8-20 \mathrm{~Hz}$, but not $4-8 \mathrm{~Hz}$, pallidal oscillations [81]. In contrast, single unit oscillations in the cerebellar-recipient thalamus near $5 \mathrm{~Hz}$ are highly coherent with rest tremor in humans [82], and correspond temporally to tremor episodes in monkeys [83].

Neuronal oscillations are also correlated with bradykinesia and rigidity. MPTP-treated monkeys lacking the typical rest tremor of PD exhibit low frequency oscillations in single unit activity similar to those with tremor $[59,84]$. Chronic levodopa [85], combined levodopa/dopamine agonist treatment [59], and therapeutic DBS [76, 84] increase spontaneous movement and suppress these low-frequency oscillations. Higher frequency "beta" $(\sim 15-30 \mathrm{~Hz})$ oscillations are correlated with rigidity and bradykinesia in humans and rodent models of PD [86, 87] (but see [88]). Single unit and LFP beta oscillations were recorded from the STN and GPi of human with PD, and beta power reductions were correlated with clinical improvement (Fig. 2D) [87, 89-91]. Enhanced beta frequency oscillations are observed in the cortex, striatum, GP, STN, and SNr of dopamine-depleted rodents $[92,93]$. Some studies, however, found that enhanced neuronal oscillations emerge after motor deficits in the dopamine-deficient state $[63,68,94]$, arguing against a causal role for "pathologic" oscillations in the genesis of bradykinesia and rigidity.

Levodopa-induced dyskinesias are also associated with characteristic neuronal oscillations. The STN and $\mathrm{GPi} / \mathrm{SNr}$ of dyskinetic humans and rats show elevated LFP power at low frequencies $(5-10 \mathrm{~Hz})$ [95-97] contralateral to the dyskinetic, but not nondyskinetic, hemibody $[98,99]$. As rats became dyskinetic, a shift from beta to high gamma $(\sim 70-100 \mathrm{~Hz})$ oscillations was observed in the striatum [100]. A similar betato-high gamma shift is observed in the STN and GPi of levodopa-treated humans, though no mention was made of whether the patients experienced dyskinesias (Fig. 2D) [89]. It should be noted that high gamma oscillations are also observed in nondyskinetic healthy rats treated with apomorphine or amphetamine [101].

\section{Synchronization}

"Synchrony" describes two or more events that occur nearly simultaneously, and may refer to single unit spikes, LFP phase across recording sites, or consistent relationships between single unit spikes and LFP phase. The LFP itself represents coordinated transmembrane potential fluctuations, and is thus a measure of local synchrony. The PD literature often refers to "burst synchrony" or "oscillatory synchrony" as if these concepts are inseparable. However, not all bursts occur in an oscillatory pattern, tonically (nonburst) firing neurons may fire synchronously, and not all synchronized activity is periodic (Fig. 2C) [102]. For example, GPe neurons exhibit more nonoscillatory synchrony than GPi neurons after MPTP [59] (Fig. 2C).

Under normal physiologic conditions in awake animals, BG and motor cortical neurons rarely fire synchronously [103]. After dopamine depletion, however, single unit oscillations in the GPi, GPe, and STN become highly synchronized with each other (Fig. 2B) $[4,59,68,84,92,104]$. TAN and GPi oscillations are also synchronized in dopamine-depleted primates [80]. DBS [84] and chronic combined levodopa/dopamine agonist treatment [59] tend to reduce interneuronal synchrony. Despite the strong evidence for a role of single unit synchrony in the pathophysiology of PD, however, at least one study found that synchrony develops after motor deficits [63]. On the other hand, in the BG-recipient thalamus, synchrony increased before clinical parkinsonism became evident [62]. 
Related to synchrony is the loss of specificity of BG neurons for passive and active movement [105]. The proportion of neurons with firing rate changes during movement is larger in MPTP treated monkeys compared to controls [106], and an increased proportion of BG and thalamic neurons encode for movement about multiple joints $[62,107]$. Collectively, these data suggest that information transfer into and out of the BG is compromised in the dopamine-depleted BG.

LFP oscillations throughout cortico-BG-thalamic circuits also synchronize after dopamine depletion [108]. The exaggerated beta oscillations of the dopamine-deficient state are highly coherent across the cortex and BG [68, 93]. Single unit activity is also highly entrained to "pathologic" LFPs [68, 90, 93, 109].

\section{DISCUSSION}

How the observed changes in cortico-BG-thalamic circuits described above translate into the motor features of PD remains a central question in PD research. Here, we discuss the relationship of the available data to the pathophysiology of PD and key areas for future investigation.

\section{The relationship of chemical dopamine depletion to $P D$}

The 6-OHDA and MPTP models are quite good at modeling the physiologic and behavioral effects of dopamine loss. Nonetheless, a couple of caveats are required. First, the toxins act quickly, creating rapid dopamine loss that is clearly different from the slow progression of PD. Second, MPTP and 6-OHDA selectively kill monoaminergic neurons, but do not recapitulate the progressive degeneration of all neuronal types (e.g., cholinergic) affected by PD [110]. With these issues in mind, the clinical similarities to human PD (including therapeutic responses) argue that these models capture the dopa-responsive motor features of PD and their physiologic correlates well [2].

\section{The origins of "pathologic" physiology}

Because of the looped nature of cortical-BGthalamic circuits, it is difficult to distinguish cause from effect among the many changes observed in the dopamine-depleted state. Here, we start with the striatum to suggest a sequence of events that could lead to electrophysiological and clinical parkinsonism.
Consistent with the "rate" model, iMSN firing rates in anesthetized rats increase after dopamine depletion [20]. Because striatopallidal synapses exhibit short-term facilitation, rapid iMSN firing may drive pause-and-burst activity in GPe [111]. This is an attractive hypothesis, as it explains how selectively driving iMSNs can cause akinesia [54] and excessive BG bursting. In essence, information may be translated from a rate code in the striatum to a pattern code in GPe. This hypothesis should be directly testable by selectively driving/identifying dMSNs and iMSNs with optogenetic techniques while recording from their target regions. Microanatomic changes in the striatum and GPe (see Functional Anatomy) also likely contribute to the excessive synchrony and loss of somatotopy observed in the parkinsonian state [22, 32].

The GPe-STN network has been proposed to generate pathologic rhythms (Fig. 1B, circuit 1) [24]. Bursting GPe neurons may hyperpolarize STN neurons, which fire rebound bursts that stimulate GPe and perpetuate the cycle. The dopamine-denervated STN may also be intrinsically more "bursty" [24]. In support of the importance of a GPe-STN oscillator in the generation of pathologic rhythms, local pharmacologic manipulations [53] and lesions [81] of the GPe and STN decrease oscillations and bursting at the BG output. Modeling studies indicate that the GPe-STN oscillator may be capable of generating beta rhythms [112], though it may not be able to do so in isolation [113]. Alternatively, aberrant cortical activity could drive pathologic activity in the STN via the hyperdirect pathway, as suggested by reduced oscillatory activity after local blockade of glutamate receptors in the STN [53]. The GPe-STN circuit is well positioned to transmit pathological patterns to the BG output given its divergent and potent projections to these nuclei.

The rate model predicts that excessive BG output should suppress thalamic firing, which does not seem to be the case [62]. Instead, pallidal output may influence the fine timing of thalamic spikes driven by cortical inputs [114], and/or gate cortico-cortical communication by regulating transitions between burst ("closed" gate) and tonic ("open" gate) firing modes [47]. Whether excessive/bursty GPi activity forces bursting in thalamocortical neurons remains to be determined.

Given the role of thalamocortical circuits in generating oscillatory synchrony in sensory systems [45], it is highly likely that they play a role in the generation of pathologic rhythms in PD. This may occur at a network level in the reciprocal loops between the cortex and thalamus (Fig. 1C, circuits 2 and 3), or within local 
motor cortical circuits (Fig. 1C, circuit 4) [115]. Compared to single spikes, thalamocortical bursts evoke long lasting, spatially distributed activity [116], suggesting that synchronized thalamocortical bursts could drive an intracortical beta resonance. These possibilities should be testable in awake, behaving animals using selective optogenetic manipulation of thalamocortical neurons.

Plastic changes in cortical-BG-thalamic circuits clearly play a role in the circuit-level physiology of PD [19, 21, 22, 26, 32, 42]. Beta oscillations and synchrony emerge with chronic, but not acute, decreases in dopamine signaling [63, 68, 94]. Further, motor deficits develop progressively after dopamine loss/receptor blockade [42], presumably due to altered dopaminergic and cholinergic regulation of cortico- and thalamostriatal synaptic plasticity. Synaptic plasticity may also be influenced by burst-firing both pre- and postsynaptically to the bursting neuron [117].

Finally, the BG influence motor output via direct connections to brainstem nuclei including the PPN and SC $[34,35]$. The PPN has been explored as a DBS site for dopa-refractory gait disturbances, albeit with mixed results [118]. It projects throughout the neuraxis, but of particular relevance to $P D$ are its reciprocal connections with the BG output nuclei, projections to intralaminar thalamus, and descending projections to the spinal cord [118]. Thus, the PPN gives the BG direct access to the "final common pathway," and also creates yet another cortical-BG-thalamic loop. It has been difficult to study in animal models, however, because it comprises multiple cell types, lacks clear borders, and its functions probably vary substantially across species [35].

\section{The relationship between "pathologic" physiology and clinical parkinsonism}

Determining how network-level changes relate to clinical parkinsonism remains an elusive goal. No doubt many of these changes have direct causal links with distinct motor abnormalities, but it is equally likely that some do not.

Parkinsonian tremor is an excellent illustration of this problem [119]. The cerebellar recipient thalamus exhibits oscillations that are highly correlated with tremor [82, 83], and DBS or ablation of this region [120] is very effective for parkinsonian tremor. The cerebellar recipient thalamus is therefore a likely tremor generator, but is not directly connected to the BG (Fig. 1C). It is not obvious how striatal dopamine loss is translated into tremor, or why DRT, anticholinergics, and STN/GPi DBS effectively suppress tremors.
Subthalamo-cerebellar connections (Fig. 1C) link the $\mathrm{BG}$ and cerebellum, but do not readily explain the efficacy of GPi DBS. Alternatively, the BG-recipient thalamus may influence the cerebellar recipient thalamus through recurrent thalamocortical connections [119].

Beta oscillations are consistently correlated with bradykinesia and rigidity (but not tremor). Transient cortical and BG beta oscillations also occur in healthy subjects, in whom they are associated with resistance to altering the current motor plan $[86,121]$. This may normally allow movements to be completed without distraction, but, if not appropriately terminated (possibly by cholinergic or dopaminergic mechanisms), persistent beta may prevent initiation of new actions [101, 121, 122]. From a mechanistic standpoint, this behavioral inertia could be related to excessive entrainment of single units throughout the motor system to a stable beta state $[68,93,101,121]$. This hypothesis implies that beta oscillations cause bradykinesia and rigidity by enhancing neuronal synchrony, which reduces the information that can be transmitted by neuronal ensembles [123]. In PD, this may be reflected in imprecise movements or rigidity as small groups of muscles are no longer activated independently.

Insight into how aberrant neural activity causes parkinsonism can also be gleaned from other BG disorders. For example, dystonia shares many physiologic features with PD. Both disorders exhibit enhanced neuronal oscillations, synchrony, and burst-firing in the STN [124] and GPi [105, 125-127]. Thus, none of these changes in and of themselves can drive parkinsonism. In dystonia, however, firing rates are lower [125-127] as are peak oscillation frequencies $[95,97,126]$.

Levodopa-induced dyskinesias share many electrophysiological features with dystonia. In both cases, low frequency $(\sim 4-10 \mathrm{~Hz})$ LFP and single unit oscillations are prominent at the $\mathrm{BG}$ output and firing rates are relatively low [57, 59, 98, 99]. Burst-firing may also be increased during dyskinesias, though results are inconsistent $[58,70,71]$. The dystonic dyskinesias often observed during the onset and offset of levodopa action ("diphasic dyskinesias") may reflect an intermediate physiologic state between "on" and "on with dyskinesias". Teasing out the physiology of dyskinesias is difficult, however, as only a handful of studies have compared the "on" and "on with dyskinesias" states $[59,99]$.

\section{Concluding remarks}

Driven by the advent of DBS, the availability of animal models, and technological advances, a 
Table 1

Selected areas for future research

\begin{tabular}{ll}
\hline Systems physiology & - The relationship between neuropathology (e.g., dopamine loss) and changes in network physiology \\
& - The role of microanatomic changes after dopamine loss in generating changes in network physiology \\
& - The relationship between changes in firing rates, bursting, oscillations, and synchrony \\
& - The relationship between bradykinesia, tremor, and rigidity and changes in network activity \\
& - The roles of the "patch" and "matrix" components of striatum \\
& - The role of the "motor" thalamus \\
& - The variable response of tremor to dopamine replacement \\
& - Mechanisms of tremor responsiveness to anticholinergics \\
Tremor & - The efficacy of STN/GPi/VIM DBS for even non-dopa-responsive tremor \\
& - The clinical similarity between DBS and focal lesions \\
& - The differential effects of STN DBS and GPi DBS provoking and improving dyskinesias, respectively \\
DBS & - The correlation between dopa-responsive symptoms and DBS responsive symptoms (except for tremor) \\
& - The physiology of being "on" versus "on with dyskinesias" \\
& - The physiological differences between "diphasic" and "peak-dose" dyskinesias \\
\hline Dyskinesias &
\end{tabular}

tremendous amount of data have been collected regarding the physiologic consequences of dopamine loss. While these data have spawned numerous theories regarding the pathophysiology of $\mathrm{PD}$, the only consensus is that the "standard" model is incomplete. Now that the clinical and electrophysiological phenomenology of PD are well-described, the next steps will be to test hypotheses linking neuropathology with physiological changes, and physiological changes with clinical symptoms. This will require a range of models, taking advantage of cell-type specific manipulations available in rodents (e.g., optogenetics) and the close homology between humans and non-human primates. Many important questions remain (see the nonexhaustive list in Table 1) whose answers may not only directly impact patient care, but also elucidate the normal functions of the BG. It remains to be seen which line(s) of inquiry will provide the critical insights that allow the rational manipulation of neural circuits to achieve the ultimate goal of transforming "pathological" neural activity into patterns compatible with normal motor, cognitive, and emotional function.

\section{ACKNOWLEDGMENTS}

The authors would like to thank Drs. Bill Dauer and Roger Albin for valuable comments and discussion. Dr. Leventhal was supported by the University of Michigan and NINDS K08 NS072183.

\section{CONFLICT OF INTERESTS}

The authors have no conflict of interest to report.

\section{REFERENCES}

[1] Dauer W, \& Przedborski S (2003) Parkinson's disease: Mechanisms and models, Neuron, 39, 889-909.

[2] Cenci MA, Whishaw IQ, \& Schallert T (2002) Animal models of neurological deficits: How relevant is the rat? Nat Rev Neurosci, 3, 574-579.

[3] Blandini F, Levandis G, Bazzini E, Nappi G, \& Armentero MT (2007) Time-course of nigrostriatal damage, basal ganglia metabolic changes and behavioural alterations following intrastriatal injection of 6-hydroxydopamine in the rat: New clues from an old model. Eur J Neurosci, 25, $397-$ 405.

[4] Rivlin-Etzion M, Elias S, Heimer G, \& Bergman H (2010) Computational physiology of the basal ganglia in Parkinson's disease. Prog Brain Res, 183, 259-273.

[5] Nambu A, Tokuno H, \& Takada M (2002) Functional significance of the cortico-subthalamo-pallidal 'hyperdirect' pathway. Neurosci Res, 43, 111-117.

[6] Mathai A, \& Smith Y (2011) The corticostriatal and corticosubthalamic pathways: Two entries, one target. So what? Front Syst Neurosci, 5, 64.

[7] Sadikot AF, \& Rymar VV (2009) The primate centromedian-parafascicular complex: Anatomical organization with a note on neuromodulation. Brain Res Bull, 78, 122-130.

[8] Mallet N, Micklem BR, Henny P, Brown MT, Williams C, Bolam JP, Nakamura KC, \& Magill PJ (2012) Dichotomous organization of the external globus pallidus. Neuron, $\mathbf{7 4}$, 1075-1086.

[9] Crittenden JR, \& Graybiel AM (2011) Basal Ganglia disorders associated with imbalances in the striatal striosome and matrix compartments. Front Neuroanat, 5, 59.

[10] Graveland GA, \& DiFiglia M (1985) The frequency and distribution of medium-sized neurons with indented nuclei in the primate and rodent neostriatum. Brain Res, 327, 307311.

[11] Tepper JM, Tecuapetla F, Koós T, \& Ibáñez-Sandoval O (2010) Heterogeneity and diversity of striatal GABAergic interneurons. Front Neuroanat, 4, 150.

[12] Kreitzer AC (2009) Physiology and pharmacology of striatal neurons. Annu Rev Neurosci, 32, 127-147.

[13] Gerfen CR, \& Surmeier DJ (2011) Modulation of striatal projection systems by dopamine. Annu Rev Neurosci, 34, 441-466. 
[14] Gittis AH, Leventhal DK, Fensterheim BA, Pettibone JR, Berke JD, \& Kreitzer AC (2011) Selective inhibition of striatal fast-spiking interneurons causes dyskinesias. $\mathrm{J} \mathrm{Neu}$ rosci, 31, 15727-15731.

[15] Schulz JM, \& Reynolds JN (2013) Pause and rebound: Sensory control of cholinergic signaling in the striatum. Trends Neurosci, 36, 41-50.

[16] Graybiel AM, Baughman RW, \& Eckenstein F (1986) Cholinergic neuropil of the striatum observes striosomal boundaries. Nature, 323, 625-627.

[17] Kawaguchi Y (1992) Large aspiny cells in the matrix of the rat neostriatum in vitro: Physiological identification, relation to the compartments and excitatory postsynaptic currents. J Neurophysiol, 67, 1669-1682.

[18] Shen W, Flajolet M, Greengard P, \& Surmeier DJ (2008) Dichotomous dopaminergic control of striatal synaptic plasticity. Science, 321, 848-851.

[19] Day M, Wang Z, Ding J, An X, Ingham CA, Shering AF, Wokosin D, Ilijic E, Sun Z, Sampson AR, Mugnaini E, Deutch AY, Sesack SR, Arbuthnott GW, \& Surmeier DJ (2006) Selective elimination of glutamatergic synapses on striatopallidal neurons in Parkinson disease models. Nat Neurosci, 9, 251-259.

[20] Mallet N, Ballion B, Le Moine C, \& Gonon F (2006) Cortical inputs and GABA interneurons imbalance projection neurons in the striatum of parkinsonian rats. J Neurosci, 26, 3875-3884.

[21] Villalba RM, \& Smith Y (2011) Differential structural plasticity of corticostriatal and thalamostriatal axo-spinous synapses in MPTP-treated Parkinsonian monkeys. J Comp Neurol, 519, 989-1005.

[22] Gittis AH, Hang GB, Ladow ES, Shoenfeld LR, Atallah BV, Finkbeiner S, \& Kreitzer AC (2011) Rapid target-specific remodeling of fast-spiking inhibitory circuits after loss of dopamine. Neuron, 71, 858-868.

[23] Minamimoto T, Hori Y, \& Kimura M (2009) Roles of the thalamic CM-PF complex-Basal ganglia circuit in externally driven rebias of action. Brain Res Bull, 78, 75-79.

[24] Bevan MD, Atherton JF, \& Baufreton J (2006) Cellular principles underlying normal and pathological activity in the subthalamic nucleus. Curr Opin Neurobiol, 16, 621-628.

[25] Rommelfanger KS, \& Wichmann T (2010) Extrastriatal dopaminergic circuits of the Basal Ganglia. Front Neuroanat, 4, 139.

[26] Fan KY, Baufreton J, Surmeier DJ, Chan CS, \& Bevan MD (2012) Proliferation of external globus pallidussubthalamic nucleus synapses following degeneration of midbrain dopamine neurons. J Neurosci, 32, 13718-13728.

[27] Bostan AC, Dum RP, \& Strick PL (2010) The basal ganglia communicate with the cerebellum. Proc Natl Acad Sci U SA, 107, 8452-8456.

[28] Bergman H, Wichmann T, Karmon B, \& DeLong MR (1994) The primate subthalamic nucleus. II. Neuronal activity in the MPTP model of parkinsonism. J Neurophysiol, 72, 507-520.

[29] Benhamou L, Bronfeld M, Bar-Gad I, \& Cohen D (2012) Globus Pallidus external segment neuron classification in freely moving rats: A comparison to primates. PLoS One, 7, e45421.

[30] Abedi PM, Delaville C, De Deurwaerdère P, Benjelloun W, \& Benazzouz A (2013) Intrapallidal administration of 6-hydroxydopamine mimics in large part the electrophysiological and behavioral consequences of major dopamine depletion in the rat. Neuroscience, 236, 289-297.

[31] Chan CS, Glajch KE, Gertler TS, Guzman JN, Mercer JN, Lewis AS, Goldberg AB, Tkatch T, Shigemoto R, Flem- ing SM, Chetkovich DM, Osten P, Kita H, \& Surmeier DJ (2011) HCN channelopathy in external globus pallidus neurons in models of Parkinson's disease. Nat Neurosci, 14, 85-92.

[32] Miguelez C, Morin S, Martinez A, Goillandeau M, Bezard E, Bioulac B, \& Baufreton J (2012) Altered pallido-pallidal synaptic transmission leads to aberrant firing of globus pallidus neurons in a rat model of Parkinson's disease. J Physiol, 590, 5861-5875.

[33] Nambu A (2007) Globus pallidus internal segment. Prog Brain Res, 160, 135-150.

[34] Zhou FM, \& Lee CR (2011) Intrinsic and integrative properties of substantia nigra pars reticulata neurons. Neuroscience, 198, 69-94.

[35] Alam M, Schwabe K, \& Krauss JK (2011) The pedunculopontine nucleus area: Critical evaluation of interspecies differences relevant for its use as a target for deep brain stimulation. Brain, 134, 11-23.

[36] Soares J, Kliem MA, Betarbet R, Greenamyre JT, Yamamoto B, \& Wichmann T (2004) Role of external pallidal segment in primate parkinsonism: Comparison of the effects of 1-methyl-4-phenyl-1,2,3,6-tetrahydropyridineinduced parkinsonism and lesions of the external pallidal segment. J Neurosci, 24, 6417-6426.

[37] Kliem MA, Maidment NT, Ackerson LC, Chen S, Smith Y, \& Wichmann T (2007) Activation of nigral and pallidal dopamine D1-like receptors modulates basal ganglia outflow in monkeys. J Neurophysiol, 98, 1489-1500.

[38] Morikawa H, \& Paladini CA (2011) Dynamic regulation of midbrain dopamine neuron activity: Intrinsic, synaptic, and plasticity mechanisms. Neuroscience, 198, 95-111.

[39] Hong S, Jhou TC, Smith M, Saleem KS, \& Hikosaka O (2011) Negative reward signals from the lateral habenula to dopamine neurons are mediated by rostromedial tegmental nucleus in primates. $J$ Neurosci, 31, 11457-11471.

[40] Fujiyama F, Sohn J, Nakano T, Furuta T, Nakamura KC, Matsuda W, \& Kaneko T (2011) Exclusive and common targets of neostriatofugal projections of rat striosome neurons: A single neuron-tracing study using a viral vector. Eur J Neurosci, 33, 668-677.

[41] Tepper JM, Martin LP, \& Anderson DR (1995) GABAA receptor-mediated inhibition of rat substantia nigra dopaminergic neurons by pars reticulata projection neurons. J Neurosci, 15, 3092-3103.

[42] Beeler JA, Frank MJ, McDaid J, Alexander E, Turkson S, Sol Bernandez M, McGehee DS, \& Zhuang X (2012) A role for dopamine-mediated learning in the pathophysiology and treatment of Parkinson's disease. Cell Rep, 2 , 1747-1761.

[43] Bodor AL, Giber K, Rovó Z, Ulbert I, \& Acsády L (2008) Structural correlates of efficient GABAergic transmission in the basal ganglia-thalamus pathway. J Neurosci, 28, 30903102.

[44] Rovó Z, Ulbert I, \& Acsády L (2012) Drivers of the primate thalamus. J Neurosci, 32, 17894-17908.

[45] Jones EG (2009) Synchrony in the interconnected circuitry of the thalamus and cerebral cortex. Ann N Y Acad Sci, 1157, 10-23.

[46] Clascá F, Rubio-Garrido P, \& Jabaudon D (2012) Unveiling the diversity of thalamocortical neuron subtypes. Eur J Neurosci, 35, 1524-1532.

[47] Rubin JE, McIntyre CC, Turner RS, \& Wichmann T (2012) Basal ganglia activity patterns in parkinsonism and computational modeling of their downstream effects. Eur $J$ Neurosci, 36, 2213-2228. 
[48] Villalba RM, Wichmann T, \& Smith Y (2013) Neuronal loss in the caudal intralaminar thalamic nuclei in a primate model of Parkinson's disease. Brain Struct Funct.

[49] Reiner A, Hart NM, Lei W, \& Deng Y. (2010) Corticostriatal projection neurons-dichotomous types and dichotomous functions. Front Neuroanat, 4, 142.

[50] Pasquereau B, \& Turner RS (2011) Primary motor cortex of the parkinsonian monkey: Differential effects on the spontaneous activity of pyramidal tract-type neurons. Cereb Cortex, 21, 1362-1378

[51] Albin RL, Young AB, \& Penney JB (1989) The functional anatomy of basal ganglia disorders. Trends Neurosci, 12, 366-375.

[52] Bergman H, Wichmann T, \& DeLong MR (1990) Reversal of experimental parkinsonism by lesions of the subthalamic nucleus. Science, 249, 1436-1438.

[53] Tachibana Y, Iwamuro H, Kita H, Takada M, \& Nambu A (2011) Subthalamo-pallidal interactions underlying parkinsonian neuronal oscillations in the primate basal ganglia. Eur J Neurosci, 34, 1470-1484.

[54] Kravitz AV, Freeze BS, Parker PR, Kay K, Thwin MT, Deisseroth K, \& Kreitzer AC (2010) Regulation of parkinsonian motor behaviours by optogenetic control of basal ganglia circuitry. Nature, 466, 622-626.

[55] Wichmann T, Bergman H, Starr PA, Subramanian T, Watts RL, \& DeLong MR (1999) Comparison of MPTP-induced changes in spontaneous neuronal discharge in the internal pallidal segment and in the substantia nigra pars reticulata in primates. Exp Brain Res, 125, 397-409.

[56] Kita H, \& Kita T (2011) Cortical stimulation evokes abnormal responses in the dopamine-depleted rat basal ganglia. $J$ Neurosci, 31, 10311-10322.

[57] Papa SM, Desimone R, Fiorani M, \& Oldfield EH (1999) Internal globus pallidus discharge is nearly suppressed during levodopa-induced dyskinesias. Ann Neurol, 46, 732-738.

[58] Boraud T, Bezard E, Bioulac B, \& Gross CE (2001) Dopamine agonist-induced dyskinesias are correlated to both firing pattern and frequency alterations of pallidal neurones in the MPTP-treated monkey. Brain, 124, 546-557.

[59] Heimer G, Rivlin-Etzion M, Bar-Gad I, Goldberg JA, Haber SN, \& Bergman H (2006) Dopamine replacement therapy does not restore the full spectrum of normal pallidal activity in the 1-methyl-4-phenyl-1,2,3,6-tetra-hydropyridine primate model of Parkinsonism. J Neurosci, 26, 8101-8114.

[60] Raz A, Vaadia E, \& Bergman H (2000) Firing patterns and correlations of spontaneous discharge of pallidal neurons in the normal and the tremulous 1-methyl-4-phenyl1,2,3,6-tetrahydropyridine vervet model of parkinsonism. $J$ Neurosci, 20, 8559-8571.

[61] Goldberg JA, Boraud T, Maraton S, Haber SN, Vaadia E, \& Bergman H (2002) Enhanced synchrony among primary motor cortex neurons in the 1-methyl-4-phenyl-1,2,3,6tetrahydropyridine primate model of Parkinson's disease. J Neurosci, 22, 4639-4653.

[62] Pessiglione M, Guehl D, Rolland AS, François C, Hirsch EC, Féger J, \& Tremblay L (2005) Thalamic neuronal activity in dopamine-depleted primates: Evidence for a loss of functional segregation within basal ganglia circuits. $\mathrm{J} \mathrm{Neu}$ rosci, 25, 1523-1531.

[63] Leblois A, Meissner W, Bioulac B, Gross CE, Hansel D, \& Boraud T (2007) Late emergence of synchronized oscillatory activity in the pallidum during progressive Parkinsonism. Eur J Neurosci, 26, 1701-1713.

[64] Hahn PJ, Russo GS, Hashimoto T, Miocinovic S, Xu W, McIntyre CC, \& Vitek JL (2008) Pallidal burst activity dur- ing therapeutic deep brain stimulation. Exp Neurol, 211, 243-251.

[65] Llinás RR, \& Steriade M (2006) Bursting of thalamic neurons and states of vigilance. J Neurophysiol, 95, 3297-3308.

[66] Wichmann T, \& Soares J (2006) Neuronal firing before and after burst discharges in the monkey basal ganglia is predictably patterned in the normal state and altered in parkinsonism. J Neurophysiol, 95, 2120-2133.

[67] Breit S, Bouali-Benazzouz R, Popa RC, Gasser T, Benabid AL, \& Benazzouz A (2007) Effects of 6-hydroxydopamineinduced severe or partial lesion of the nigrostriatal pathway on the neuronal activity of pallido-subthalamic network in the rat. Exp Neurol, 205, 36-47.

[68] Mallet N, Pogosyan A, Sharott A, Csicsvari J, Bolam JP, Brown P, \& Magill PJ (2008) Disrupted dopamine transmission and the emergence of exaggerated beta oscillations in subthalamic nucleus and cerebral cortex. J Neurosci, 28, 4795-4806.

[69] Hutchinson WD, Levy R, Dostrovsky JO, Lozano AM, \& Lang AE (1997) Effects of apomorphine on globus pallidus neurons in parkinsonian patients. Ann Neurol, 42, 767-775.

[70] Merello M, Balej J, Delfino M, Cammarota A, Betti O, \& Leiguarda R (1999) Apomorphine induces changes in GPi spontaneous outflow in patients with Parkinson's disease. Mov Disord, 14, 45-49.

[71] Levy R, Dostrovsky JO, Lang AE, Sime E, Hutchison WD, \& Lozano AM (2001) Effects of apomorphine on subthalamic nucleus and globus pallidus internus neurons in patients with Parkinson's disease. J Neurophysiol, 86, 249-260.

[72] Vitek JL, Zhang J, Hashimoto T, Russo GS, \& Baker KB (2012) External pallidal stimulation improves parkinsonian motor signs and modulates neuronal activity throughout the basal ganglia thalamic network. Exp Neurol, 233, 581-586.

[73] Dorval AD, Russo GS, Hashimoto T, Xu W, Grill WM, \& Vitek JL (2008) Deep brain stimulation reduces neuronal entropy in the MPTP-primate model of Parkinson's disease. J Neurophysiol, 100, 2807-2818.

[74] Cleary DR, Raslan AM, Rubin JE, Bahgat D, Viswanathan A, Heinricher MM, \& Burchiel KJ (2013) Deep brain stimulation entrains local neuronal firing in human globus pallidus internus. J Neurophysiol, 109, 978-987.

[75] McCairn KW, \& Turner RS (2009) Deep brain stimulation of the globus pallidus internus in the parkinsonian primate: Local entrainment and suppression of low-frequency oscillations. J Neurophysiol, 101, 1941-1960.

[76] Xu W, Russo GS, Hashimoto T, Zhang J, \& Vitek JL (2008) Subthalamic nucleus stimulation modulates thalamic neuronal activity. J Neurosci, 28, 11916-11924.

[77] Magnin M, Morel A, \& Jeanmonod D (2000) Single-unit analysis of the pallidum, thalamus and subthalamic nucleus in parkinsonian patients. Neuroscience, 96, 549-564.

[78] Molnar GF, Pilliar A, Lozano AM, \& Dostrovsky JO (2005) Differences in neuronal firing rates in pallidal and cerebellar receiving areas of thalamus in patients with Parkinson's disease, essential tremor, and pain. J Neurophysiol, 93, 3094-3101.

[79] Chen MT, Morales M, Woodward DJ, Hoffer BJ, \& Janak PH (2001) In vivo extracellular recording of striatal neurons in the awake rat following unilateral 6-hydroxydopamine lesions. Exp Neurol, 171, 72-83.

[80] Raz A, Frechter-Mazar V, Feingold A, Abeles M, Vaadia E Bergman H. (2001) Activity of pallidal and striatal tonically active neurons is correlated in mptp-treated monkeys but not in normal monkeys. J Neurosci, 21, RC128 
[81] Wichmann T, Bergman H, \& DeLong MR (1994) The primate subthalamic nucleus. III. Changes in motor behavior and neuronal activity in the internal pallidum induced by subthalamic inactivation in the MPTP model of parkinsonism. J Neurophysiol, 72, 521-530.

[82] Lenz FA, Tasker RR, Kwan HC, Schnider S, Kwong R, Murayama Y, Dostrovsky JO, \& Murphy JT (1988) Single unit analysis of the human ventral thalamic nuclear group: Correlation of thalamic "tremor cells" with the 3$6 \mathrm{~Hz}$ component of parkinsonian tremor. J Neurosci, 8 , 754-764.

[83] Guehl D, Pessiglione M, Fraçois C, Yelnik J, Hirsch EC, Féger J, \& Tremblay L (2003) Tremor-related activity of neurons in the 'motor' thalamus: Changes in firing rate and pattern in the MPTP vervet model of parkinsonism. Eur $J$ Neurosci, 17, 2388-2400.

[84] Meissner W, Leblois A, Hansel D, Bioulac B, Gross CE, Benazzouz A, \& Boraud T (2005) Subthalamic high frequency stimulation resets subthalamic firing and reduces abnormal oscillations. Brain, 128, 2372-2382.

[85] Gilmour TP, Lieu CA, Nolt MJ, Piallat B, Deogaonkar M, \& Subramanian T (2011) The effects of chronic levodopa treatments on the neuronal firing properties of the subthalamic nucleus and substantia nigra reticulata in hemiparkinsonian rhesus monkeys. Exp Neurol, 228, 53-58.

[86] Jenkinson N, \& Brown P (2011) New insights into the relationship between dopamine, beta oscillations and motor function. Trends Neurosci, 34, 611-618.

[87] Kühn AA, Tsui A, Aziz T, Ray N, Brücke C, Kupsch A, Schneider GH, \& Brown P (2009) Pathological synchronisation in the subthalamic nucleus of patients with Parkinson's disease relates to both bradykinesia and rigidity. Exp Neurol, 215, 380-387.

[88] McConnell GC, So RQ, Hilliard JD, Lopomo P, \& Grill WM (2012) Effective deep brain stimulation suppresses low-frequency network oscillations in the basal ganglia by regularizing neural firing patterns. J Neurosci, 32, 15657 15668.

[89] Brown P, Oliviero A, Mazzone P, Insola A, Tonali P, \& Di Lazzaro V (2001) Dopamine dependency of oscillations between subthalamic nucleus and pallidum in Parkinson's disease. J Neurosci, 21, 1033-1038.

[90] Weinberger M, Mahant N, Hutchison WD, Lozano AM, Moro E, Hodaie M, Lang AE, \& Dostrovsky JO (2006) Beta oscillatory activity in the subthalamic nucleus and its relation to dopaminergic response in Parkinson's disease. $J$ Neurophysiol, 96, 3248-3256.

[91] Ray NJ, Jenkinson N, Wang S, Holland P, Brittain JS, Joint C, Stein JF, \& Aziz T (2008) Local field potential beta activity in the subthalamic nucleus of patients with Parkinson's disease is associated with improvements in bradykinesia after dopamine and deep brain stimulation. Exp Neurol, 213, 108-113.

[92] Mallet N, Pogosyan A, Márton LF, Bolam JP, Brown P, \& Magill PJ (2008) Parkinsonian beta oscillations in the external globus pallidus and their relationship with subthalamic nucleus activity. J Neurosci, 28, 14245-14258.

[93] Brazhnik E, Cruz AV, Avila I, Wahba MI, Novikov N, Ilieva NM, McCoy AJ, Gerber C, \& Walters JR (2012) Statedependent spike and local field synchronization between motor cortex and substantia nigra in hemiparkinsonian rats. J Neurosci, 32, 7869-7880.

[94] Degos B, Deniau JM, Chavez M, \& Maurice N (2009) Chronic but not acute dopaminergic transmission interruption promotes a progressive increase in cortical beta frequency synchronization: Relationships to vigilance state and akinesia. Cereb Cortex, 19, 1616-1630.

[95] Silberstein P, Kühn AA, Kupsch A, Trottenberg T, Krauss JK, Wöhrle JC, Mazzone P, Insola A, Di Lazzaro V, Oliviero A, Aziz T, \& Brown P (2003) Patterning of globus pallidus local field potentials differs between Parkinson's disease and dystonia. Brain, 126, 2597-2608.

[96] Meissner W, Ravenscroft P, Reese R, Harnack D, Morgenstern R, Kupsch A, Klitgaard H, Bioulac B, Gross CE, Bezard E, \& Boraud T (2006) Increased slow oscillatory activity in substantia nigra pars reticulata triggers abnormal involuntary movements in the 6-OHDA-lesioned rat in the presence of excessive extracellular striatal dopamine. Neurobiol Dis, 22, 586-598.

[97] Weinberger M, Hutchison WD, Alavi M, Hodaie M, Lozano AM, Moro E, \& Dostrovsky JO (2012) Oscillatory activity in the globus pallidus internus: Comparison between Parkinson's disease and dystonia. Clin Neurophysiol, 123, 358-368.

[98] Foffani G, Ardolino G, Meda B, Egidi M, Rampini P, Caputo E, Baselli G, \& Priori A (2005) Altered subthalamo-pallidal synchronisation in parkinsonian dyskinesias. J Neurol Neurosurg Psychiatry, 76, 426-428.

[99] Alonso-Frech F, Zamarbide I, Alegre M, Rodríguez-Oroz MC, Guridi J, Manrique M, Valencia M, Artieda J, \& Obeso JA (2006) Slow oscillatory activity and levodopa-induced dyskinesias in Parkinson's disease. Brain, 129, 1748-1757.

[100] Halje P, Tamtè M, Richter U, Mohammed M, Cenci MA, \& Petersson P (2012) Levodopa-induced dyskinesia is strongly associated with resonant cortical oscillations. JNeurosci, 32, 16541-16551.

[101] Berke JD (2009) Fast oscillations in cortical-striatal networks switch frequency following rewarding events and stimulant drugs. Eur J Neurosci, 30, 848-859.

[102] Chan V, Starr PA, \& Turner RS (2011) Bursts and oscillations as independent properties of neural activity in the parkinsonian globus pallidus internus. Neurobiol Dis, 41, $2-10$.

[103] Bar-Gad I, Heimer G, Ritov Y, \& Bergman H (2003) Functional correlations between neighboring neurons in the primate globus pallidus are weak or nonexistent. J Neurosci, 23, 4012-4016.

[104] Bergman H, Feingold A, Nini A, Raz A, Slovin H, Abeles M, \& Vaadia E (1998) Physiological aspects of information processing in the basal ganglia of normal and parkinsonian primates. Trends Neurosci, 21, 32-38.

[105] Bronfeld M, \& Bar-Gad I (2011) Loss of specificity in Basal Ganglia related movement disorders. Front Syst Neurosci, 5, 38.

[106] Leblois A, Meissner W, Bezard E, Bioulac B, Gross CE, \& Boraud T (2006) Temporal and spatial alterations in GPi neuronal encoding might contribute to slow down movement in Parkinsonian monkeys. Eur J Neurosci, 24, 1201-1208.

[107] Boraud T, Bezard E, Bioulac B, \& Gross CE (2000) Ratio of inhibited-to-activated pallidal neurons decreases dramatically during passive limb movement in the MPTP-treated monkey. J Neurophysiol, 83, 1760-1763.

[108] Dejean C, Nadjar A, Le Moine C, Bioulac B, Gross CE, \& Boraud T (2012) Evolution of the dynamic properties of the cortex-basal ganglia network after dopaminergic depletion in rats. Neurobiol Dis, 46, 402-413.

[109] Goldberg JA, Rokni U, Boraud T, Vaadia E, \& Bergman $\mathrm{H}$ (2004) Spike synchronization in the cortex/basal-ganglia networks of Parkinsonian primates reflects global dynamics of the local field potentials. J Neurosci, 24, 6003-6010. 
[110] Braak H, Del Tredici K, Rüb U, de Vos RA, Jansen Steur EN, \& Braak E (2003) Staging of brain pathology related to sporadic Parkinson's disease. Neurobiol Aging, 24, 197-211.

[111] Kim J, \& Kita H (2013) Short-term plasticity shapes activity pattern-dependent striato-pallidal synaptic transmission. J Neurophysiol, 109, 932-939.

[112] Holgado AJ, Terry JR, \& Bogacz R (2010) Conditions for the generation of beta oscillations in the subthalamic nucleusglobus pallidus network. J Neurosci, 30, 12340-12352.

[113] Stanford IM, Loucif KC, Wilson CL, Cash D, \& Lacey MG (2005) Limitations of the Isolated GP-STN Network In The Basal Ganglia VIII, Bolam JP, Ingham CA, \& Magill PJ, eds. Springer Science, New York, NY, pp. 65-73.

[114] Goldberg JH, \& Fee MS (2012) A cortical motor nucleus drives the basal ganglia-recipient thalamus in singing birds. Nat Neurosci.

[115] Castro-Alamancos MA (2013) The motor cortex: A network tuned to 7-14 Hz. Front Neural Circuits, 7, 21.

[116] Beierlein M, Fall CP, Rinzel J, \& Yuste R (2002) Thalamocortical bursts trigger recurrent activity in neocortical networks: Layer 4 as a frequency-dependent gate. $\mathrm{J} \mathrm{Neu-}$ rosci, 22, 9885-9894.

[117] Sjöström PJ, Rancz EA, Roth A, \& Häusser M (2008) Dendritic excitability and synaptic plasticity. Physiol Rev, 88, 769-840.

[118] Hamani C, Moro E, \& Lozano AM (2011) The pedunculopontine nucleus as a target for deep brain stimulation. J Neural Transm, 118, 1461-1468.

[119] Helmich RC, Hallett M, Deuschl G, Toni I, \& Bloem BR (2012) Cerebral causes and consequences of parkinsonian resting tremor: A tale of two circuits? Brain, 135, 32063226.

[120] Benabid AL, Pollak P, Gao D, Hoffmann D, Limousin P, Gay E, Payen I, \& Benazzouz A (1996) Chronic electrical stimulation of the ventralis intermedius nucleus of the thalamus as a treatment of movement disorders. J Neurosurg, 84, 203-214.

[121] Leventhal DK, Gage GJ, Schmidt R, Pettibone JR, Case AC, \& Berke JD (2012) Basal Ganglia Beta oscillations accompany cue utilization. Neuron, 73, 523-536.

[122] McCarthy MM, Moore-Kochlacs C, Gu X, Boyden ES, Han X, \& Kopell N. (2011) Striatal origin of the pathologic beta oscillations in Parkinson's disease. Proc Natl Acad Sci $U S A$.

[123] Averbeck BB, Latham PE, \& Pouget A (2006) Neural correlations, population coding and computation. Nat Rev Neurosci, 7, 358-366.

[124] Schrock LE, Ostrem JL, Turner RS, Shimamoto SA, \& Starr PA (2009) The subthalamic nucleus in primary dystonia: Single-unit discharge characteristics. J Neurophysiol, 102, 3740-3752.

[125] Vitek JL, Chockkan V, Zhang JY, Kaneoke Y, Evatt M, DeLong MR, Triche S, Mewes K, Hashimoto T, \& Bakay RA (1999) Neuronal activity in the basal ganglia in patients with generalized dystonia and hemiballismus. Ann Neurol, 46, 22-35

[126] Starr PA, Rau GM, Davis V, Marks WJ, Ostrem JL, Simmons D, Lindsey N, \& Turner RS (2005) Spontaneous pallidal neuronal activity in human dystonia: Comparison with Parkinson's disease and normal macaque. J Neurophysiol, 93, 3165-3176.

[127] Tang JK, Moro E, Mahant N, Hutchison WD, Lang AE, Lozano AM, \& Dostrovsky JO (2007) Neuronal firing rates and patterns in the globus pallidus internus of patients with cervical dystonia differ from those with Parkinson's disease. J Neurophysiol, 98, 720-729. 